\title{
Egreso seguro en cirugía mayor ambulatoria: 2,886 pacientes
}

\author{
Safe discharge in an ambulatory surgery unit: 2886 patients \\ Omar Alberto Paipilla Monroy,* Jorge Luis Green Solís,* César Romero Mejía*
}

\footnotetext{
Palabras clave: Cirugía mayor ambulatoria, egreso seguro, evaluación postoperatoria.

Key words: Major ambulatory surgery, secure discharge, postoperative evaluation.
}

* Unidad de Especialidades Médicas de Baja California, en Tijuana (UNEME).

Recibido: 17/03/2016 Aceptado: 26/07/2016

\section{RESUMEN}

Introducción: En las últimas décadas, han ocurrido cambios en los servicios de salud, destacando la inclusión de la cirugía mayor ambulatoria. Esta modalidad de atención se acompaña de varios beneficios: altera en grado mínimo el modo de vida del paciente y sus familiares, el enfermo recibe atención más personal, su ansiedad se atenúa, los costos se reducen, entre otros. Objetivo: Valorar un instrumento (hoja de control) que permita el egreso seguro del paciente o que señale si debe ser trasladado a otra unidad. Material y métodos: Estudio prospectivo en la Unidad de Especialidades Médicas de Tijuana, Baja California, del $1^{\circ}$ de agosto de 2007 al 31 de diciembre de 2009. Se consideraron las personas que aceptaron la cirugía mayor ambulatoria y que cumplieron los criterios que señala la Norma Oficial Mexicana NOM-205-SSA1-2002. Se usó una hoja de valoración, diseñada para calificar el egreso seguro del paciente. Resultados: Fueron incluidos 2,886 pacientes, 1,908 mujeres y 978 hombres. En los procedimientos quirúrgicos predominaron la colecistectomía laparoscópica (754 casos) y las resecciones/biopsias de tumores de tejidos blandos (505 casos). El 11.6\% de los pacientes presentó enfermedad concomitante, siendo la hipertensión arterial y la diabetes mellitus las de mayor frecuencia. El $44 \%$ de los pacientes egresó entre la primera y la cuarta hora de su ingreso al área de recuperación, el 36\% egresó entre la cuarta y la octava hora, y sólo el $20 \%$ requirió más de ocho horas para egresar. No hubo defunciones. Veintinueve pacientes requirieron traslado al hospital. Conclusión: Los resultados son satisfactorios. Con el empleo de una hoja de control de datos en el postoperatorio inmediato se pudo calificar de manera segura a los pacientes que pueden ser egresados pocas horas después de la cirugía.

\section{ABSTRACT}

Introduction: In the last decades, there have been changes in health services, standing out the inclusion of ambulatory major surgery. This mode of care is accompanied by several benefits: it minimally alters the way of life of the patient and his relatives, the patient receives more personal attention, his anxiety is attenuated, costs are reduced, among others. Objective: To evaluate an instrument (control sheet) that allows the safe discharge of the patient or to indicate if it should be transferred to another unit. Material and methods: Prospective study at the Medical Specialties Unit of Tijuana, Baja California, from August 1, 2007 to December 31, 2009. The individuals who accepted the ambulatory major surgery and who met the criteria set forth in Official Mexican Standard NOM-205-SSA1-2002 were considered. A score sheet, designed to rate the patient's safe discharge, was used. Results: 2,886 patients, 1,908 women and 978 men were included. In the surgical procedures, predominated laparoscopic cholecystectomy (754 cases) and resections/biopsies of soft tissue tumors (505 cases). $11.6 \%$ of the patients presented concomitant disease, with hypertension and diabetes mellitus being the most frequent. $34 \%$ of the patients were released between the first and fourth hours of admission to the recovery area, $36 \%$ were released between the fourth and eighth hours, and only $20 \%$ required more than eight hours to be released. There were no deaths. Twenty-nine patients required transfer to the hospital. Conclusion: The results are satisfactory. With the use of a data control sheet in the immediate postoperative period, it was possible to safely rate patients who may be discharged within a few hours after surgery.

\section{INTRODUCCIÓN}

$\mathrm{E}_{\mathrm{b}}^{\mathrm{n}}$ n las últimas décadas ha ocurrido un cambio importante en los servicios de salud, destacando la inclusión de la cirugía mayor ambulatoria, que se ha convertido en un pa- radigma de la asistencia médica. El Instituto de Seguridad Social y Servicios Sociales de los Trabajadores del Estado (ISSSTE) emitió en 1976 una guía que señaló las normas de esta cirugía y los procedimientos a realizarse; sin embargo, no se implantó de manera amplia. ${ }^{1}$ 
En 2014, el Comité de Elaboración de Guías de Práctica Clínica de la Asociación Mexicana de Cirugía General, AC presentó las bases del tratamiento ambulatorio en cirugía mayor para obtener los mejores resultados. ${ }^{2}$

En 2005 se publicó en el Periódico Oficial de Baja California el decreto de creación de la Unidad de Especialidades Médicas de Baja California (UNEME); se inauguró la primera en todo el país en Ensenada, y en 2007 la de Tijuana, que funciona de las siete de la mañana a las ocho de la noche, cuando se cierra la unidad. ${ }^{3}$

Esta modalidad de atención se acompaña de una serie de beneficios, más allá de los económicos, que en gran medida han facilitado su difusión y crecimiento. ${ }^{4}$ Altera en grado mínimo el modo de vida del paciente y sus familiares, el enfermo recibe una atención más personal, su ansiedad se atenúa, los costos se reducen, disminuye el riesgo de infección hospitalaria, existe menor incapacidad laboral y un retorno temprano a las actividades habituales, asimismo descienden los diferimientos de cirugía en los hospitales generales y unidades de tercer nivel. Hace más de diez años, el danés H. Kehlet publicó el texto que en España se editó como Fast track surgery: realidades y dificultades. ${ }^{5}$ En nuestro país, las comunicaciones al respecto han sido limitadas. ${ }^{6}$

Objetivo: valorar un instrumento (hoja de control) que permita un egreso seguro del individuo o que sea una alarma que señale que el sujeto no puede egresar en forma segura o debe ser trasladado a otra unidad para su atención. ${ }^{7}$

\section{MATERIAL Y MÉTODOS}

Se realizó un estudio prospectivo en la UNEME Tijuana en el periodo comprendido del $1 .^{\circ}$ de agosto de 2007 al 31 de diciembre de 2009. Se definió como "cirugía mayor ambulatoria" (CMA) aquella en la que se realizan procedimientos quirúrgicos que no exigen que el enfermo ingrese antes de la operación y que permiten que después de la misma sea egresado antes de 24 horas; que haya recibido cualquier tipo de anestesia y requiera de un periodo de recuperación u observación postoperatoria; contrasta con la cirugía menor ambulatoria, que incluye a los procedimientos realizados con anestesia local, que no requieren vigilancia postoperatoria ni estancia hospitalaria.

Se incluyeron las personas que aceptaron CMA. Se utilizó la Norma Oficial Mexicana NOM-205-SSA1-2002 para definir la práctica quirúrgica en nuestra institución. ${ }^{7}$ Seleccionamos los 22 criterios de inclusión señalados por dicha norma. El paciente debía tener un expediente clínico completo, con valoración anestésica y de la especialidad involucrada, estado físico I a II de la clasificación American Society of Anesthesiology (ASA) y haber sido programado por su cirujano. El índice de masa corporal (IMC) pudo ser hasta 35 si el individuo fue intervenido con anestesia regional; se aceptó hasta 40 si se usó anestesia local más sedación, pero quedó a criterio del anestesiólogo si fue mayor de 40. En la consulta externa, el 100\% de los sujetos aceptaron su inclusión a nuestro programa ambulatorio. Se excluyeron los que tenían expediente incompleto, así como los sometidos a procedimientos de corta estancia y que requirieron traslado al Hospital General, y aquellos con diagnóstico postquirúrgico distinto al preoperatorio; también fueron excluidos los que tuvieron complicaciones, pues en ellos no se pudo aplicar la evaluación para egreso ambulatorio.

Como parte del protocolo de la unidad, estaba señalada la administración profiláctica de antibiótico en todas aquellas personas que recibieron algún material protésico, así como la administración preoperatoria de analgésicos (ketorolaco), antieméticos (ondansetrón) y dexametasona; la finalidad de administrar esta fue potenciar el efecto del ondansetrón. También se administraron inhibidores de la bomba de protones.

En recuperación se usó una hoja diseñada para calificar el egreso seguro del paciente; la evaluación contenía cinco parámetros: signos vitales (presión arterial y pulso), nivel de actividad (ambulación), dolor (moderado o intenso), tolerancia a la vía oral (náusea o vómito), hemorragia (por la incisión o drenaje) (Figura 1). Cada parámetro tenía una calificación de cero, uno o dos; el dos indicaba "ausencia o normalidad", el uno traducía "moderado", y el cero fue "severo o incapacitante". Se anotaron las evaluaciones cada hora y cuando menos debía existir la suma de nueve puntos en dos registros consecutivos para considerar el egreso del individuo. 


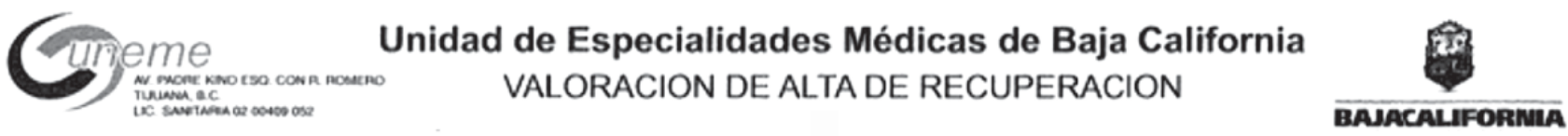

Hora en que llega a recuperación.

$10: 35$

\section{SIGNOS VITALES}

Presion Anterial y pulso dentro de limites de $20 \%$ de cilra basal prooperatoria Presión Arterial y pulso, en limites de $20-40 \%$ de ctra basal preoperatoria Presión Avterial y pulso, mayores do 40\% de la cifra basal preoperatoria

NIVEL DE ACTIVIDAD

Marcha segura, sin mareos, similar a la del preoperasorio

Nocesita ayuda para levantarse

Incapaz de caminar

\section{DOLOR}

Dolor minimo o aceptable

Dolor inaceptable, intenso

\section{NAUSEAS Y VOMITOS}

Minimo: desaparecen satisfactoriamente o con medicamentos

Moderados: desaparecen con medicamentos

Intensos: persisten aùn y despuess de repetir tratamiento

\section{HEMORRAGIA POR LA INCISION}

Minimo no necesita cambios de apositos

Moderado incluso necesta dos carnbios de apositos

Imtonso: se nocositan más de tres cambios de apositos

El paciente debe contar con un minimo de 9 purtos para su alta

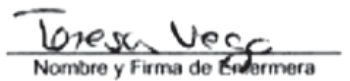

Figura 1. Hoja de valoración alta.

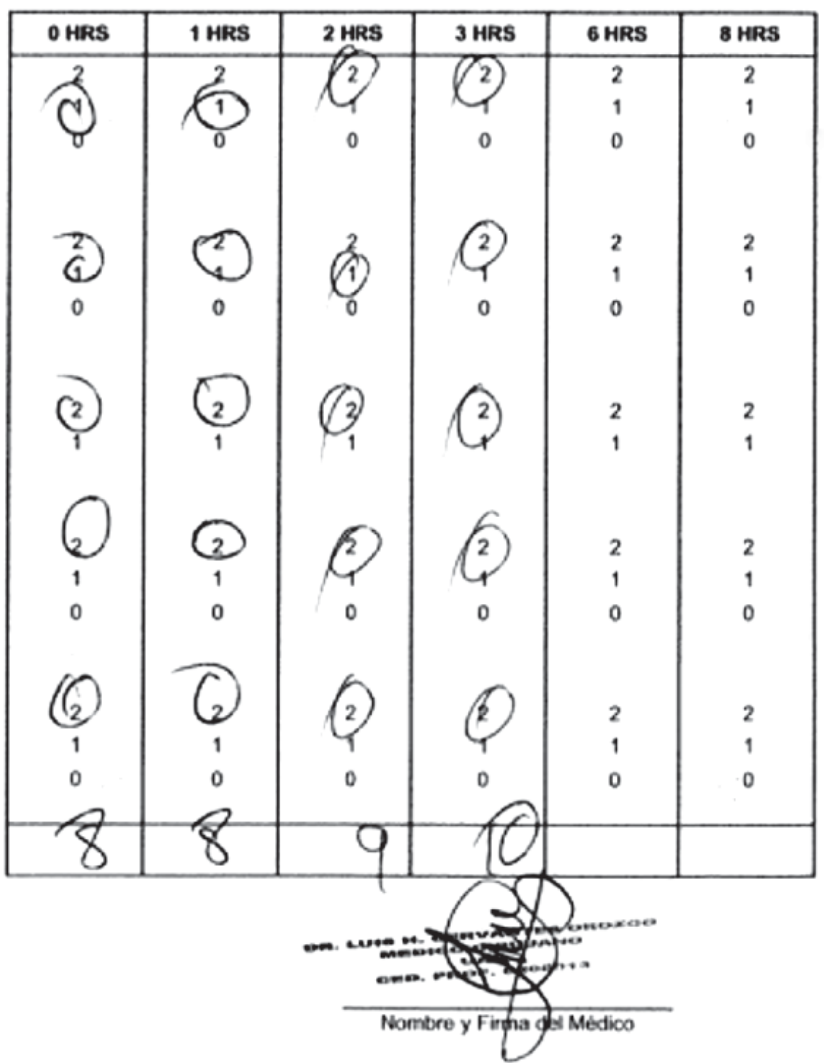

Toda la información se recolectó de manera sistemática en una planilla diseñada en Excel, y la información se evaluó para su análisis y discusión.

\section{RESULTADOS}

Fueron incluidos 2,886 sujetos: 1,908 mujeres y 978 hombres, con edad promedio de 34 años, a quienes se les realizaron los siguientes procedimientos: colecistectomía laparoscópica (754), resección/biopsia de tumores de tejidos blandos (505), obstrucción tubárica bilateral laparoscópica (407), plastía inguinal (320), cirugía oftalmológica (223), cirugía ortopédica
(147), amigdalectomía (136), plastía umbilical (130), hemorroidectomía y fistulectomía (92), safenectomía (40), plastía de pared abdominal con malla (36), septumplastía (36), ooforectomía laparoscópica (29), queiloplastía (18) y procedimientos endoscópicos (13). Encontramos enfermedad concomitante en el $11.6 \%$ de las personas, siendo la hipertensión arterial y la diabetes mellitus las de mayor frecuencia. El IMC promedio fue de 27; el tiempo quirúrgico promedio, 40 minutos; el promedio de recuperación anestésica inmediata fue 49.6 minutos y el de estancia postquirúrgica fue 5.33 horas.

En nuestra unidad, la totalidad de las evaluaciones y el llenado de las hojas fueron realizados 
por el personal de enfermería adscrito a recuperación. El 19\% de los pacientes tuvo nueve puntos o más en la primera hora de ingreso; el 25\% cumplió con criterio de egreso de la segunda a la cuarta hora de vigilancia. El 36\% egresó entre la cuarta y la octava hora, y sólo el $20 \%$ requirió más de ocho horas para obtener el criterio de egreso. No hubo defunciones. Con el fin de determinar si ingresaron individuos al Hospital General, se revisaron los libros del servicio de urgencias y se encontró lo siguiente:

Dieciocho casos de colecistectomía laparoscópica. Cinco se ingresaron por fuga biliar, la resolución fue laparotomía y colocación de sonda en T. Se presentó un sujeto con lesión de la vía biliar que se reparó ocho semanas después del evento. Seis enfermos tuvieron hematoma; se llevó a cabo abordaje laparoscópico, se lavó y se dejó drenaje. Seis personas reportaron dolor, al cual se le dio manejo por 24 horas y se egresaron.

Una paciente en quien se realizó obstrucción tubaria bilateral presentó dolor y se reexploró; se encontró hematoma retroperitoneal contenido con lesión de arteria iliaca, la cual se reparó.

Siete individuos de plastía inguinal se reingresaron por hematoma; dos de ellos requirieron orquiectomía y el resto, drenaje del hematoma y lavado mecánico.

De aquellos en quienes se llevó a cabo plastía de pared, se ingresaron dos para manejo de dolor por 48 horas y se egresaron.

Un sujeto con septumplastía se ingresó por epistaxis.

En seis casos pediátricos hubo sangrado postamigdalectomía que solo requirió observación y egreso posterior.

Laparotomía exploradora por sangrado que llevó a choque a una persona en la que se hizo ooforectomía laparoscópica.

Cuatro pacientes de queiloplastía presentaron espasmos respiratorios; hubo necesidad de nebulización para su manejo.

\section{DISCUSIÓN}

Desde 1960 se iniciaron los procedimientos de cirugía ambulatoria mayor; poco a poco fueron aumentando las comunicaciones con intervenciones cada vez más complejas, lo cual permitió que en algunos países como Estados Unidos de
Norteamérica se realizaran hasta $68 \%$ de los casos que previamente eran hospitalizados; esto, actualmente, sigue en aumento. ${ }^{8-13}$

En México también existe un interés que sigue la tendencia mundial, pero no se cuenta con el número de unidades específicas para atender a aquellos individuos que desde el preoperatorio se supone que serán de cirugía ambulatoria estricta.

Las causas fundamentales del crecimiento de esta técnica han sido la adecuada selección de enfermos, la protocolización del proceso asistencial desde la admisión de sujetos hasta su egreso, los avances tecnológicos y farmacológicos que permiten realizar técnicas anestésicas y quirúrgicas seguras con una recuperación más rápida, la actitud positiva de los profesionales de la salud hacia este nuevo modelo de trabajo, así como la alta satisfacción de las personas con la atención recibida. Se debe señalar la conveniencia en reducir la estancia hospitalaria que satura los servicios de cirugía y la disminución del costo, que ha resultado significante. De este modo, cada vez se exploran procedimientos quirúrgicos que puedan ser incluidos en las listas autorizadas de pacientes candidatos a cirugía mayor ambulatoria.

\section{CONCLUSIÓN}

Nuestros resultados son satisfactorios y tanto los individuos como las autoridades médicas aprecian esta conducta, la cual crece cada día. También los cirujanos involucrados prefieren la técnica, que permite reducir la ocupación hospitalaria y el tiempo de espera de los sujetos para ser atendidos. Las enfermeras capacitadas para el cuidado de las personas que reciben una intervención quirúrgica y son candidatas a egreso temprano realizan, asimismo, una extraordinaria labor en su vigilancia y registro. En cirugía mayor, de 2,886 pacientes incluidos en esta revisión no ocurrieron defunciones y sólo 29 fueron enviados al hospital.

Con el empleo de una hoja de almacén de datos en el postoperatorio inmediato aplicada por el anestesiólogo, el cirujano y la enfermera, hemos podido calificar de manera segura a los individuos que pueden ser egresados en menos de seis horas. 


\section{REFERENCIAS}

1. Alvarado GFJ, Vega SE. La cirugía ambulatoria, una opción para mejorar la calidad de la atención. Experiencia de 15 años. Revista de Especialidades MédicoQuirúrgicas, Instituto de Seguridad Social y Servicios Sociales de los Trabajadores del Estado, México. 2006; 11: 34-37.

2. Comité de elaboración de Guías de Práctica Clínica. Guía de Práctica Clínica. Cirugía mayor ambulatoria. México, DF: Asociación Mexicana de Cirugía AC; 2014.

3. Decreto del Ejecutivo por el cual se aprueba la Creación de la Unidad de Especialidades Médicas de Baja California. Periódico Oficial del Estado de Baja California, Tomo CXII. Mexicali, Baja California; 18 de marzo de 2005.

4. American Society of Anesthesiologists. Practice guidelines for postanesthetic care. A report by the American Society of Anesthesiologists Task Force on postanesthetic care. Anesthesiology 2002; 96: 742-752.

5. Kehlet H. Fast track surgery: realidades y dificultades. Cir Esp. 2006; 80: 187-188.

6. Guzmán EH. Unidades de Cirugía Ambulatoria. Curso de postgrado $1.38 .^{\circ}$ Congreso Internacional de Cirugía General. León, Gto., Mex. Asociación Mexicana de Cirugía General; 2014. pp. 44

7. Norma Oficial Mexicana NOM-205-SSA 1-2002. Para la práctica de la cirugía ambulatoria mayor. Diario Oficial de la Federación. 22 de Junio 2004.
8. Schaefer MK1, Jhung M, Dahl M, Schillie S, Simpson C, Llata E, et al. Infection control assessment of ambulatory surgical centers. JAMA. 2010; 303: 2273-2279.

9. Acevedo A, León J. Ambulatory hernia surgery under local anesthesia is feasible and safe in obese patients. Hernia. 2010; 14: 57-62.

10. Chung F, Assmann N. Car accidents after ambulatory surgery in patients without an escort. Anesth Analg. 2008; 106: 817-820.

11. Bueno LJ, Granero CP, Gomez IG, Ibañez CJ, López AR, García GE. Twenty-five years of ambulatory laparoscopic cholecystectomy. Cir Esp. 2016; 94: 429-441.

12. Al-Omani S, Almodhaiberi $\mathrm{H}$, Ali B, Alballa A, Alsowaina K, Alhasan I, et al. Feasibility and safety of day-surgery laparoscopic cholecystectomy: a singleinstitution 5-year experience of 1140 cases. Korean J Hepatobiliary Pancreat Surg. 2015; 19: 109-112.

13. Cabrera EY, Cifuentes PA, Sanabria A, Domínguez LC. Tiroidectomía ambulatoria: análisis de minimización de costos en Colombia. Rev Colomb Cir. 2014; 29: 319-326.

Correspondencia: Dr. Omar Alberto Paipilla Monroy Calle. Joaquín Clausel 10343-5, Zona Río Tijuana, 22320, Tijuana, Baja California, México.

E-mail:drpaipilla@hotmail.com 